\title{
Discharge Characteristics of Nano-sized Electrode with Aligned Carbon Nanotubes Grown on Tungsten Whisker Tip under Various Gas Conditions
}

\author{
Bo Liang, Akihisa Ogino, and Masaaki Nagatsu \\ Graduate School of Science and Technology, Shizuoka University \\ 3-5-1 Johoku, Naka-ku, Hamamatsu 432-8561, Japan
}

(Received

)

\begin{abstract}
:
Vertically aligned multiwalled carbon nanotubes were grown on a tungsten (W) tip by thermal chemical vapor deposition (CVD), applying a negative biasing voltage to the W tip in a pin-plate electrodes configuration. The carbon nanotube(CNT) electrodes, due to their nano-sized tip radii, create very high electric fields, and reduce significantly the breakdown voltage. Comparing the CNTs-grown $\mathrm{W}$ tip electrode to the $\mathrm{W}$ tip electrode, the ignition voltage for the former case decreased from $230 \mathrm{~V}$ to $181 \mathrm{~V}$, while the saturation current increased from $12 \mu \mathrm{A}$ to $20 \mu \mathrm{A}$. These results are attributed to a relatively high field enhancement factor $\beta \sim 960$ of the CNTs electrodes compared to $\beta \sim 60$ for the simple $\mathrm{W}$ tip. There are also significant differences between the $\mathrm{W}$ tip electrodes with and without CNTs in the values of the igniting voltage and discharge current, when the $\mathrm{W}$ tips were used as cathode.
\end{abstract}

PACS: 52.80.-s, 52.90.+z, 81.07.-b

KEYWORDS: Tungsten tip, Carbon nanotube, Nano-sized electrode, Field emission 


\section{Introduction}

Recently, micro-sized plasmas have attracted much attention in various industrial fields,

such as ionized wind cooling, thin film coating, and surface modification [1-6]. A variety of electrode configurations including cylinders, slits, micro-tubes and micro-slots have been used. However, there are some issues of those silicon or metal electrode materials presented in those papers. One of the problems is that these materials cannot simultaneously meet the requirements of having a good thermal stability and also a high electric conductivity as electrode material. So, preventing the silicon or copper electrodes at corners and tips from being melted or even broken due to a long discharging period is an issue that remains to be solved. Another one is the expansion/contraction of the electrode during discharge/charge cycling, which leads to pulverization and causes capacity loss. Moreover, developing a technology to overcome these problems would also improve other aspects such as the possibility to decrease the breakdown voltage, minimize the power supply system, and reduce the cost of the electrode fabricating process. These are very important when it comes to applications. Carbon nanotubes $[7,8]$ with the excellent physical properties, such as good thermal and electric conductivity [9, 10], stable emission current $[10,11]$, and long life time $[12,13]$ are promising materials for electron sources and electrodes.

So far, there have been few reports of presenting the gas discharges using nano-sized 
electrodes. The objective in the present study is to investigate the characteristics of nano-sized discharges using aligned carbon nanotubes(CNTs) grown on tungsten(W) tip electrode under various gas conditions, aiming at developing the future ultra-fine material processing technologies, such as etching or deposition.

Differently from micro-plasmas produced using parallel electrodes [1-3], the pin-plate electrodes were used to operate nano-scaled discharge in this paper. Due to the field enhance factor of the W tip electrode, especially in the case of forest of CNTs on its surface, it is easy to ignite discharge at lower applying voltage under the low pressure gas circumstance. Comparison between discharge characteristics using a W tip-plane electrode and a CNTs covered W tip-plane electrode was carried out under different gas species and pressures. The use of CNTs covered W tip electrode can clearly decrease breakdown voltage and increase discharge current, when it was as cathode. However, the degradation of CNTs on the W whisker was observed after a long time discharge operation by comparing to the initial value of breakdown voltage and self-sustaining discharge current.

\section{Experimental}

A pencil-like tungsten whisker was made using electrochemical etching technique, by dipping a tungsten wire (30 $\mu \mathrm{m}$ in diameter) in $\mathrm{NaOH}(1 \mathrm{~mol} / \mathrm{l})$ electrolysis solution with an 
electric bias of $13 \mathrm{~V}$ (DC) between the wire and the Titanium (Ti) electrode at an output current of $0.06 \mathrm{~A}$. The curvature radius of the tungsten tip is as small as $100 \mathrm{~nm}$, and its surface is relatively smooth. First, nickel (Ni) particles were deposited as catalyst on the apex of the $\mathrm{W}$ whisker by RF magnetron plasma processing (100 W net power) for $40 \mathrm{~s}$. The MWCNTs were grown by chemical vapor deposition (CVD) on the W whisker substrate. $[14,15]$ After ten minutes pretreatment by ammonia $\left(\mathrm{NH}_{3}\right)$ gas, the CNTs growth process was performed for 5-15 min under following conditions: the gas flow rates of $\mathrm{NH}_{3}$, and $\mathrm{C}_{2} \mathrm{H}_{2}$ were 150 , and $50 \mathrm{sccm}$, respectively, the pressure was fixed at 30 Torr, and the stage heating temperature was $900{ }^{\circ} \mathrm{C}$. A key point in obtaining vertically aligned CNTs is applying a dc voltage during the growing process [16]. In our experiment $-100 \mathrm{~V}$ dc were applied between the whisker and a Ti mesh situated at a distance of approximately $2 \mathrm{~mm}$.

Figure 1(a) shows the detailed configuration of the discharge system using various gases. A dc voltage is supplied between a flat electrode (iron plate) and the CNTs electrode. The distance between two electrodes can be precisely controlled by adjusting a micrometer head. The typical FE-SEM images of the W tip before and after CNTs growth are shown in Fig. 1(b). The CNTs grown on the $\mathrm{W}$ tip are typically 50 100 nm in diameter and about $15 \mu \mathrm{m}$ in length. The field emission characteristics of the CNTs electrode were measured using a parallel diode configuration at a pressure of $10^{-6}$ Torr, 
where the electrode separation was typically $80 \mu \mathrm{m}$. The current-voltatge discharge properties in various gases were also measured in the same geometrical conditions.

\section{Experimental Results and Discussion}

The CNTs grown on the W tip are typically 50 100 nm in diameter, as shown in Fig. 2. Zhang et al. [17] showed that SWCNTs grew in the direction of an applied electric field of the order of $1.0 \mathrm{~V} / \mu \mathrm{m}$. They also maintained that the dipole moment induced by the local electric field led to sufficiently large aligning torques and force on the CNTs. Considering the experimental result of field emission property of the $\mathrm{W}$ tip-plane electrodes, that is, it has a field enhancement factor of about 60 , the value of biasing voltage in our experiment agrees with the order of $\mathrm{V} / \mu \mathrm{m}$.

Figure 3 shows the field emission characteristics at a pressure of $10^{-6}$ Torr, i.e., the emission current (I) versus the applied voltage (V) and the Fowler-Nordheim (F-N) plots for both the cases of $\mathrm{W}$ tip without CNTs(black triangle plot) and W tip with CNTs(red circle plot). The turn-on value of the electric field for the CNTs electrode decreased to about $3.4 \mathrm{~V} / \mu \mathrm{m}$, compared to $15 \mathrm{~V} / \mu \mathrm{m}$ for $\mathrm{W}$ tip only. Assuming the work function of CNTs to be roughly $5 \mathrm{eV}$ [18], the field enhancement factor $\beta$ for the CNTs electrode is evaluated to be about $\beta \sim 960$, determined from the slope of F-N plot, whereas it is only 60 for the W tip without CNTs, as shown in Fig. 3 (b). The result in the case of CNTs 
electrode indicates a clear improvement due to the presence of the vertically aligned CNTs on the tip surface. Individual CNTs on the W tip create very high electric fields near the tips $[19,20]$, because they have a high aspect ratio $(\mathrm{h} / \mathrm{\rho})$ [21, 22], (where $\mathrm{h}$ is the height of the CNT and $\rho$ is the radius of its cap). This facilitates the breakdown process of gases between two electrodes. It is easier to ignite discharge in the case of CNTs covered W tip electrode than in the case of a pure $\mathrm{W}$ tip.

The I-V curves of a dc discharge between a W tip electrode (with and without CNTs) and a flat iron electrode were measured to investigate the effect of CNTs, in He at a fixed pressure of 10 Torr. The results are shown in Fig. 4, where the discharge was ignited at $181 \mathrm{~V}$ in the case of the CNTs covered W tip electrode as cathode, while the breakdown occurred at $230 \mathrm{~V}$ with a discharge current of $12 \mu \mathrm{A}$ in the case of $\mathrm{W}$ tip as the cathode. Using CNTs on the tip, the breakdown voltage in helium can be reduced by nearly $25 \%$, while the discharge current significantly increases from $12 \mu \mathrm{A}$ to $20 \mu \mathrm{A}$. It is expected that a great number of CNTs participate in the ionization process producing a high discharge current. The dc discharge behavior with the CNTs electrodes was also investigated in various gases. Figure 5 shows the breakdown voltages of several gases at a fixed chamber pressure of 10 Torr. In this case, another CNTs covered tip electrode different from the one used in the measurements shown in Fig. 4 was used. The physical properties of these CNTs are slightly different (shorter and lower surface density). The anode-cathode 
distance was kept at $80 \mu \mathrm{m}$ in all the cases. Note that each gas shows a distinct breakdown voltage; for example, He shows the lowest ignition voltage at about $181 \mathrm{~V}$, while ammonia the highest value, about $563 \mathrm{~V}$.

The breakdown voltage for the molecular gases, such as oxygen and air, are higher than that of argon or helium, because additional energy was required to sustain the discharge under the various elementary processes, such as dissociation of the molecules, ionization of the dissociated species and electron attachment for oxygen atoms or molecules.

It is noted that He has lower breakdown voltage than Ar, even though helium has a higher ionization potential of $24.58 \mathrm{eV}$ than argon of $15.76 \mathrm{eV}$. At $0{ }^{\circ} \mathrm{C}$ and atmospheric pressure, the electron mean free path $\lambda_{\mathrm{e}}(=173.6 \mathrm{~nm})$ in helium is a factor of 2.8 longer than that in argon ( $\left.\lambda_{\mathrm{e}}=62.6 \mathrm{~nm}\right)$. Therefore, under the same applied electric field E, electrons in helium gas can gain higher acceleration energy $E \lambda_{\mathrm{e}}$ till the next collision than those in argon. The electric field satisfying that $E \lambda_{e}$ equals ionization voltage of helium is a factor of 0.56 lower than that of argon. Therefore, it is understood that helium has lower breakdown voltage than argon in the present pin-plane electrode geometry with nonuniform electric field.

Next, we investigated the effect of the gas pressure on the breakdown voltage and discharge current in different gases. Figure 6(a) presents the pressure dependence on the 
breakdown voltage for air, ammonia, argon, helium, and nitrogen, respectively. It is interesting to note that the breakdown voltage does not significantly change and only slightly increases with gas pressure from 100 to 700 Torr. According to the previous result of F-N plot under the vacuum shown in Fig. 3, the field enhancement factor of CNTs grown $\mathrm{W}$ tip was evaluated as $\beta \sim 960$. For the applying voltage of about $250 \mathrm{~V}$ in case of helium and an inter-electrode distance $\mathrm{d}$ of $80 \mu \mathrm{m}$, an enhanced electric field is roughly estimated as $\mathrm{E} \sim 3.0 \times 10^{9} \mathrm{~V} / \mathrm{m}$. At a pressure of 700 Torr, for instance, electron mean free path $\lambda_{\mathrm{e}}$ in helium is estimated to be about $188.5 \mathrm{~nm}$. Therefore, we obtain an acceleration voltage $\mathrm{E} \lambda_{\mathrm{e}}$ between the mean free path $\lambda_{\mathrm{e}}$ as $565.5 \mathrm{~V}$, which is much higher than the ionization voltage of helium. So, even in the case of highest pressure of 700 Torr, it is expected that the electrons can gain the acceleration energy sufficient enough to ionize gas species. Here, if we simply assume that the electric field should satisfy the condition that $E \lambda_{e}$ equals the ionization voltage to sustain the discharge, neglecting the secondary emission effect in anode, then breakdown voltage $V_{B}=E d$ will simply vary in proportional to the pressure.

The discharge currents in the cases of argon, helium, nitrogen, oxygen, and ammonia are plotted as a function of gas pressure in Fig. 6(b). It is also interesting to note that the discharge currents are linearly increasing with the gas pressure. Remembering that the breakdown voltages were almost constant in the gas pressure range, as shown in Fig. 6(a), 
the resistivity of discharge is inversely proportional to the gas pressure, because the discharge currents are proportional to the pressure. Since the distance between two electrodes is fixed, the product of electrical conductivities and discharging area is supposed to be linearly proportional to the gas pressure. At present, we have no clear understanding, but there are two possible reasons for that. One possibility is that the electrical conductivity, that is, plasma density in the discharge region increased with the gas pressure. The other is due to an increase of effective cross-section of the discharge volume with the pressure. To figure out these possibilities, we will need the further investigation.

The polarity of the CNTs electrode (as anode or cathode of the discharge) plays an important role in discharge characteristics. Figures 7(a) and (b) show the real discharge pictures of positive corona (in the case of the CNTs electrode polarized as anode) and negative corona (for the CNTs electrode biased as cathode), respectively. The CNTs with a curvature radius of $50 \mathrm{~nm}$ create very high nonlinear electric fields near the tips. When the CNTs electrode is used as cathode, the electrons are easily pulled out from the tips of nanotubes by the field emission due to a high field enhancement effect and they will serve as seed electrons to create a self-sustained discharge more easily. While it is used as anode, the field emission from the plane plate cathode is not expected, so we need a higher voltage to ignite the discharge than that in the former case. Due to the same reason, a more compressive bright region with purplish red color was observed in the negative 
corona compared to the flat and large illuminant area in the positive corona. The corresponding I-V curves of Ar discharges (at a pressure of 10 Torr and electrodes distance of $80 \mu \mathrm{m}$ ), are shown in Fig. 7 (c). For the positive corona the current collected by a whisker in both cases (with and without nanotubes) strongly increases at $330 \mathrm{~V}$, then saturates at a value of about $4 \times 10^{-7} \mathrm{~A}$. In the case of negative corona, the gas ignites at 250 $\mathrm{V}$ for CNTs electrode which is roughly $60 \mathrm{~V}$ lower than the value determined for the simple tip electrode. It is considered that in the breakdown process lots of aligned nanotubes participate as electron emitters, leading to a more extensive discharge current, about 10 times higher than that measured for the simple tip electrode in the positive corona case. Therefore, the breakdown voltages are close for CNTs and W tip electrodes if the flat electrode is used as cathode, while the breakdown voltage for CNTs electrode is lower than that for $\mathrm{W}$ tip if the flat electrode is anode. To achieve a low breakdown voltage and high self-sustaining current in gases discharge, it is necessary to make CNTs as electron emitter and the flat electrode as passive collector of electrons.

As shown in Fig. 8(a), and (b), supplying a 30 cycles of 120 seconds triangular impulses with voltage from $-310 \mathrm{~V}$ to $300 \mathrm{~V}$ between two electrodes, the breakdown voltage and the maximum discharge current of each period were measured to investigate the aging effect of CNTs electrode. Figures 8(c) show the difference between the breakdown voltage values, and the maximum currents $\left(\mathrm{I}_{\mathrm{M}}\right)$ in the two cases of the tip used 
as cathode with and without CNTs. The I-V curve was measured in the voltage conditions presented above, in argon at 10 torr and having $80 \mu \mathrm{m}$ between the electrodes. It is found that in the case of the CNTs electrode (as cathode) compared to the W whisker electrode (as cathode) case the differences between the values of breakdown voltage, and also the values of $\mathrm{I}_{\mathrm{M}}$ become smaller. The breakdown voltage drops about $10 \mathrm{~V}$ compared to the initial value. For maximum current, the difference slightly decreases from $1.4 \times 10^{-5} \mathrm{~A}$ to $1 \times 10^{-5} \mathrm{~A}$. These modifications seem correlated and are probably due to the fact that the CNTs at the top of the whisk are gradually damaged by the energetic ions. The difference both in breakdown voltage and $\mathrm{I}_{\mathrm{M}}$ happens only when the CNTs and $\mathrm{W}$ tip used as cathode, and they take the role as collector of ions.

\section{Conclusion}

In this study, vertically aligned CNTs were grown on a W tip. The characteristics of the discharge using the tip as an electrode were investigated and compared to the case of a simple metallic tip. Due to the field enhancement factor of $\beta \sim 960$ of the vertically aligned CNT forest, much higher nonlinear electric field is created compare to the case of a W tip with a field enhancement factor of $\sim 60$. This hastens the breakdown process. The experimental results clearly show that the breakdown voltage was significantly reduced when the CNTs electrode was used as cathode compared to the simple tip electrode case. At 
the same time, a strong increase of the discharge current was achieved at the saturated voltage.

By changing gas species, we found the inherent discharge characteristics of breakdown voltages and current depending upon gas species. The present results, however, showed the degradation of the CNTs electrode after several repeated discharges, therefore, it will be necessary to optimize the discharge voltage and current performance to avoid the damage by improving the field emission performance of CNTs electrode.

\section{Acknowledgments}

This work was supported in part by the Grants-in-Aid for Scientific Research and performed under 21st Century COE Program "Research and Education Center of Nanovision of Science” by the Japan Society for the Promotion of Science (JSPS) and by the Research and Education Funding for Research Promotion Project, MEXT, Japan. 


\section{References}

[1] Becker K H, Schoenbach K H and Eden J G, 2006 J. Phys. D: Appl. Phys. 3955

[2] Sankaran R M and Giapia K P 2003 J.Phys.D: Appl.Phys. 362914

[3] Frame J W, Wheeler DJ, Detemple T A, and Eden J G 1997 Appl. Phys. Lett. 71 1165

[4] Tachibana K, Feng S, and Sakai T 2000 J. Appl. Phys. 884967

[5] Go D B, Garimella S V, and Fisher T S 2007 J. Appl. Phys. 102053302

[6] Hsu C P, Jewell-Larsen N E, Krichtafovitch I A, and Mamishev A V $2009 \mathrm{~J}$. Microelectromech. Syst. 18111

[7] Iijima S 1991 Nature 35456

[8] Iijima S and Ichihashi T 1993 Nature 363603

[9] Bonard J M, Salvetat J P, Stockli T, Forro L, and Chatelain A 1999 Appl. Phys. A: Mater. Sci. Process. 69245

[10]De heer W A, Chatelain A, and Ugarte D 1995 Science 2701179

[11]Ma X C, Wang E G, Zhou W Z, Jefferson D A, Deng S Z, Xu N S, and Yuan J 1999 Appl. Phys. Lett. 753105

[12]Purcell S T, Vincent P, Journet C, and Binh V T 2002 Phys. Rev. Lett. 88105502

[13]Dean K A and Chalamala B R 1999 Appl. Phys. Lett.75 3017

[14]Zhang Z, Wei B Q, Ramanath G, and Ajayan P M 2000 Appl. Phys. Lett. 773764

[15]Wei B Q, Vajtai R, Jung Y et al 2002 Nature 416495

[16] Matsuda T, Mesko M, Ishikawa T, Sato J, Ogino A, and Nagatsu M $2008 \mathrm{Jpn}$. J. Appl. Phys. 477436

[17]Zhang Y, Chang A, Cao J, Wang Q, Kim W, Li Y, Morris N, Yenilmez E, Kong J, and Dai H 2001 Appl. Phys. Lett. 793155

[18]Gao R, Pan Z, Wang Z L 2001 Appl.Phys.Lett. 781757

[19]De heer W A, Chatelain A and Ugarte D 1995 Science 2701179

[20]Forbes R G, Edgcombe CJ and Valdre U 2003 Ultramicroscopy 9557

[21]Kokkotskis G C, Modinos A, Xanthakis J P 2002 J. Appl. Phys. 914580

[22]Kokkotskis G C, Roumelistis J A, Xanthakis J P 2004 J. Appl. Phys. 951468 


\section{Figure Captions}

Fig. 1 (a)Schematic drawing of discharging system, and (b) FE-SEM images of a W whisker before (left) and after (right) CNTs growth.

Fig. 2 FE-SEM image of MWCNTs on the W tip (left) and TEM image of a single MWCNT in the forest of CNTs.

Fig. 3 (a)Field emission characteristics of emission current versus the applied electric field, and (b)Fowler-Nordheim plots for both the cases of W tip electrode (black triangle) and the CNTs electrode (red circle).

Fig. 4 The I-V curves of the CNTs electrode (red solid circle) and W tip electrode (black solid square) as cathode $80 \mu \mathrm{m}$ away from the iron plate anode under the same experimental conditions.

Fig. 5 The I-V curves for various gases at pressure of 10 Torr.

Fig. 6 Gas pressure dependencies of (a) the breakdown voltage of various gases, and (b) the discharge currents at saturated voltage.

Fig. 7 Photos of discharges when the CNTs was used as (a) anode and (b) cathode in argon at 10 Torr with the electrodes separation of $80 \mu \mathrm{m}$. (c) The I-V curves of positive corona phase(left) and negative corona phase(right), respectively.

Fig. 8 Aging effects of the CNTs electrode: (a) the triangular impulses with voltage from $-310 \mathrm{~V}$ to $300 \mathrm{~V}$ in a cycle, and (b) the corresponding I-V curve using it as cathode. (c) The breakdown voltages and the maximum currents of CNTs electrode (as cathode) and W tip electrode (as cathode) recorded in 30 cycles. 


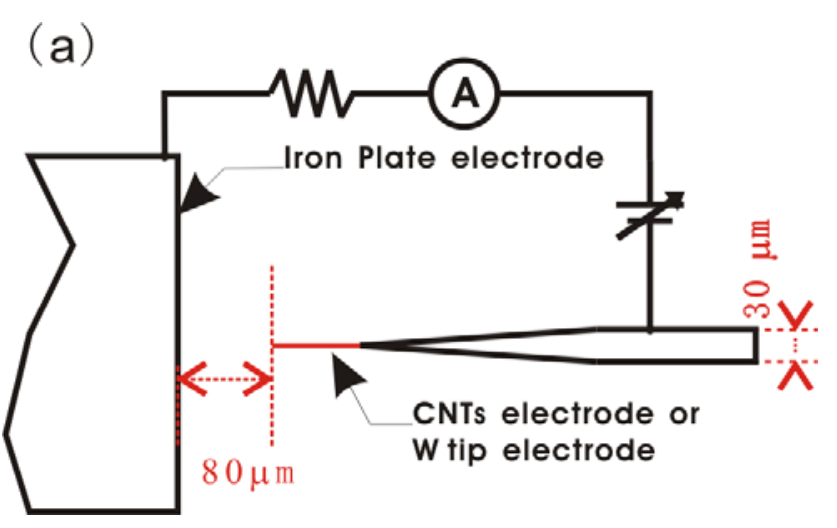

Figure 1(a) 


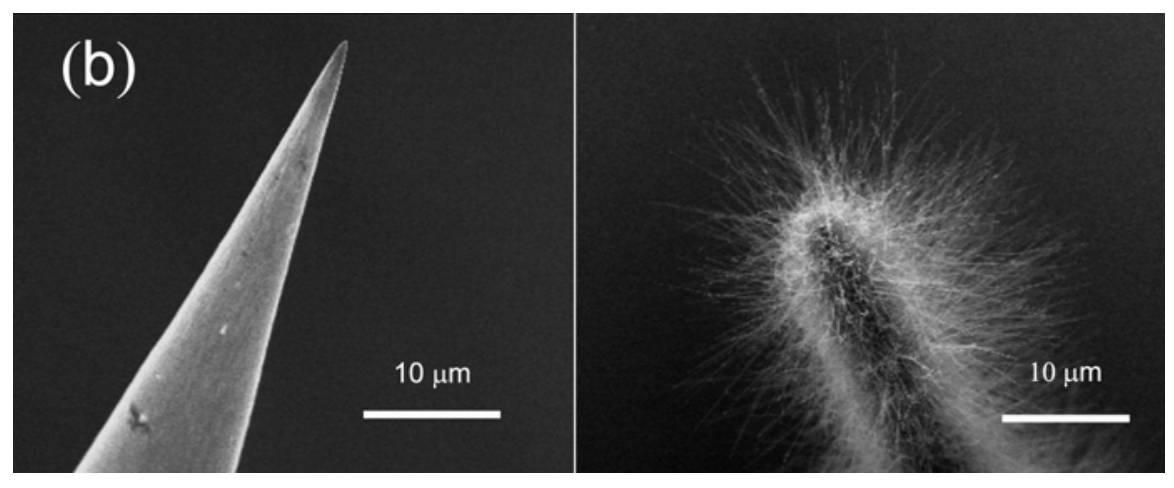

Figure 1(b) 


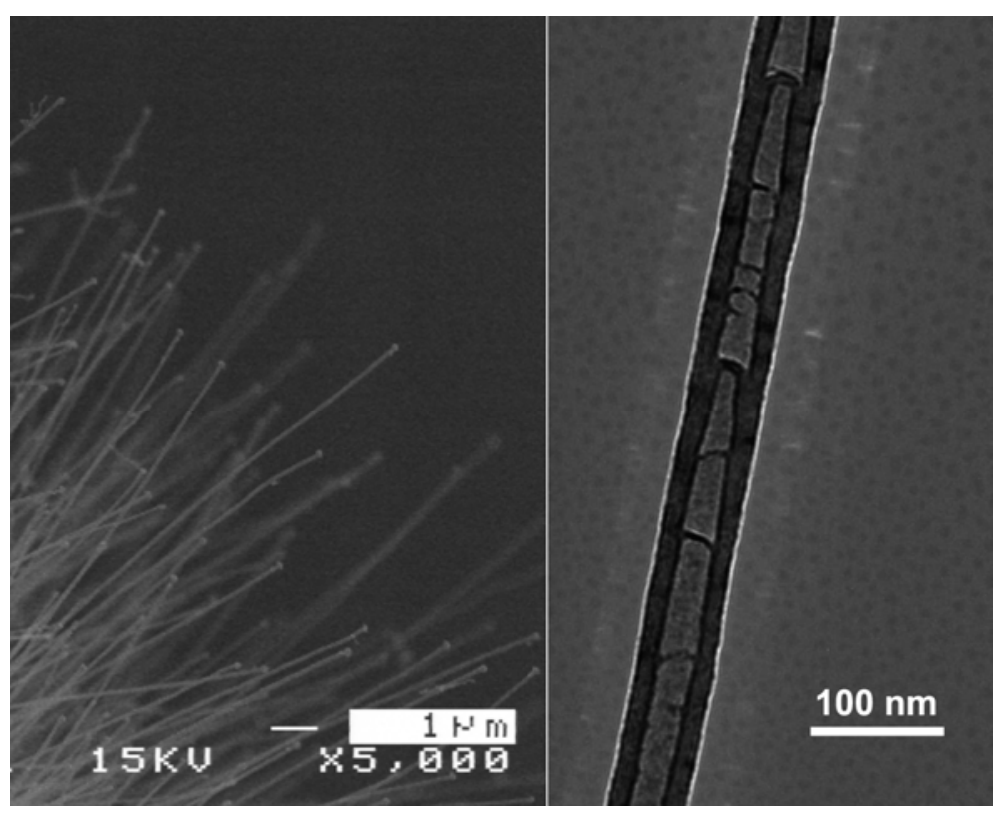

Figure 2 


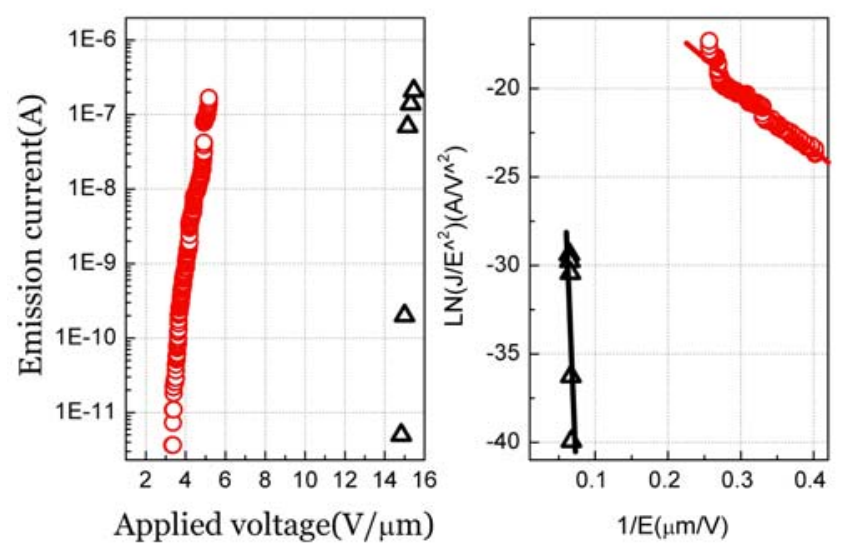

Figure 3 


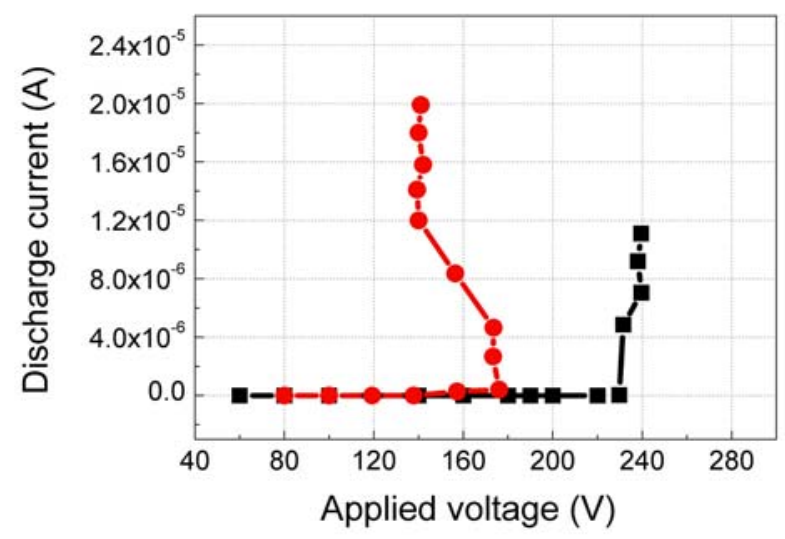

Figure 4 


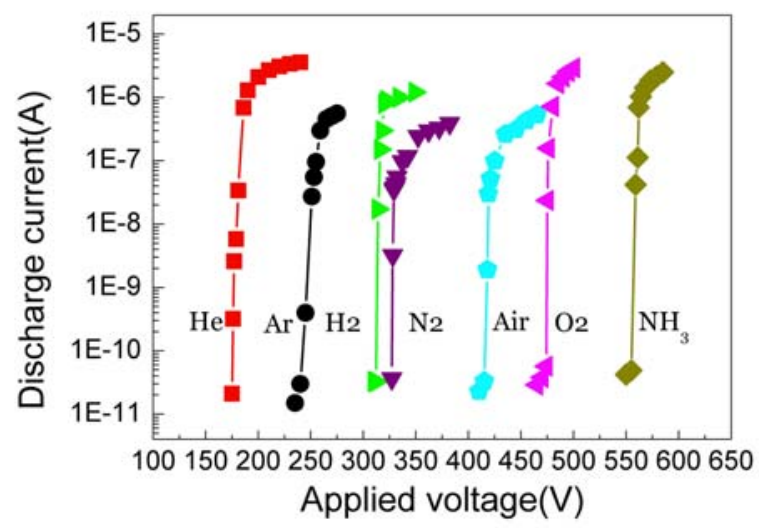

Figure 5 
(a)

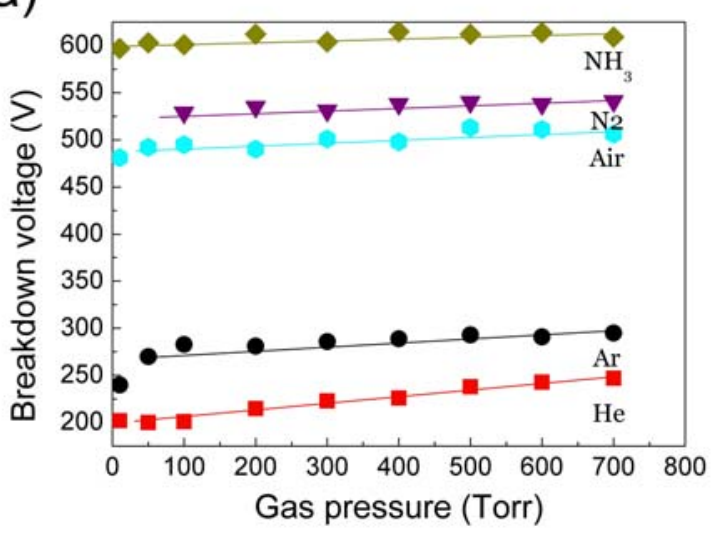

Figure 6(a) 
(b)

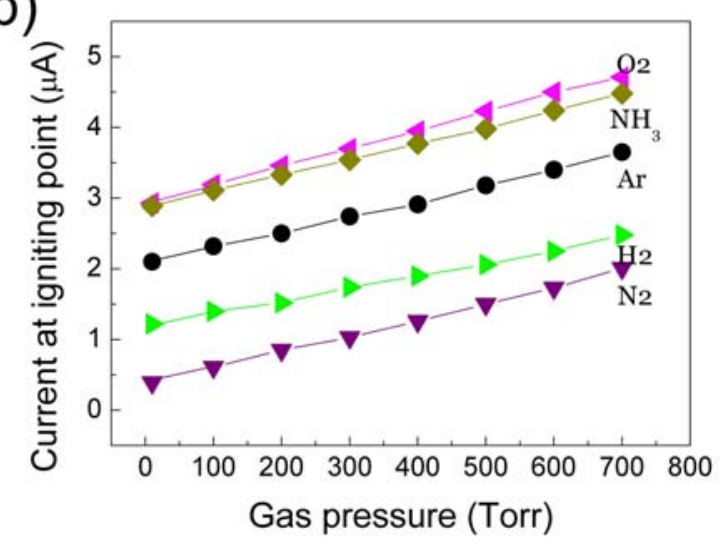

Figure 6(b) 


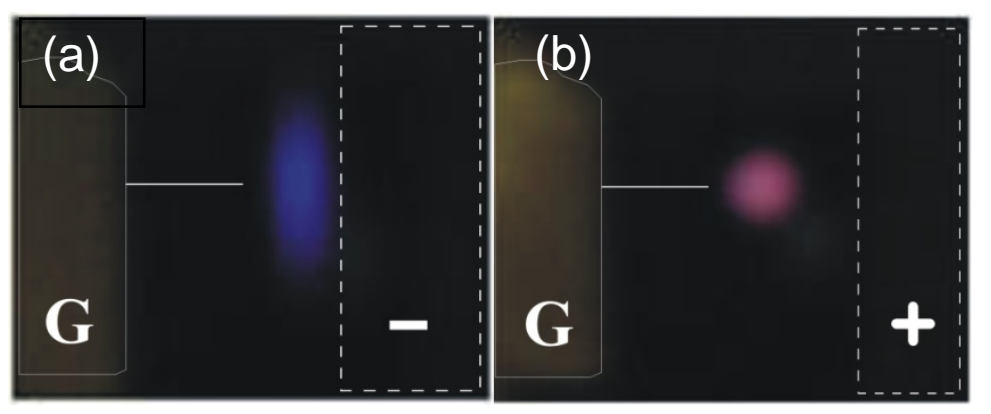

Figure 7(a),(b) 
(c)

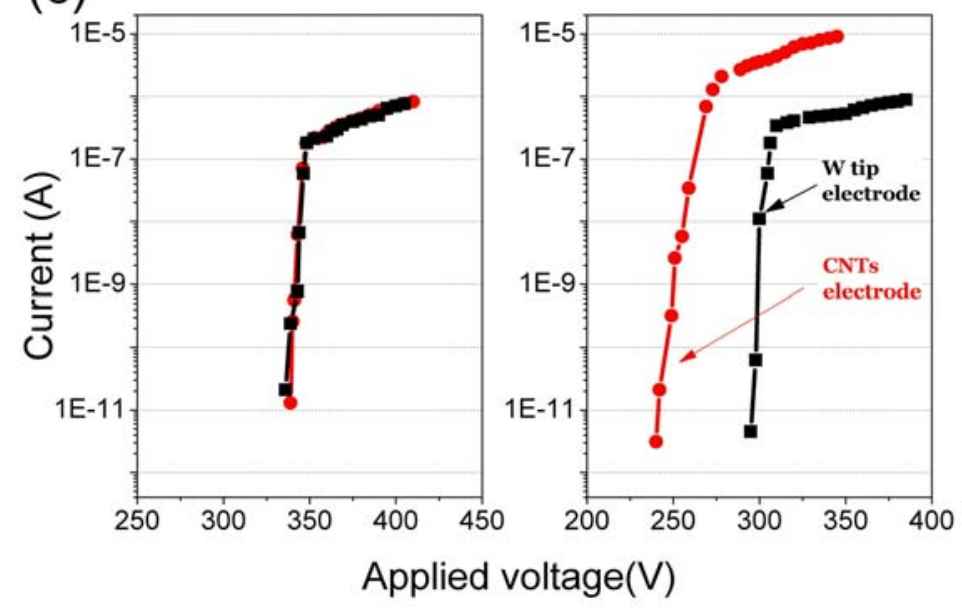

Figure 7(c) 


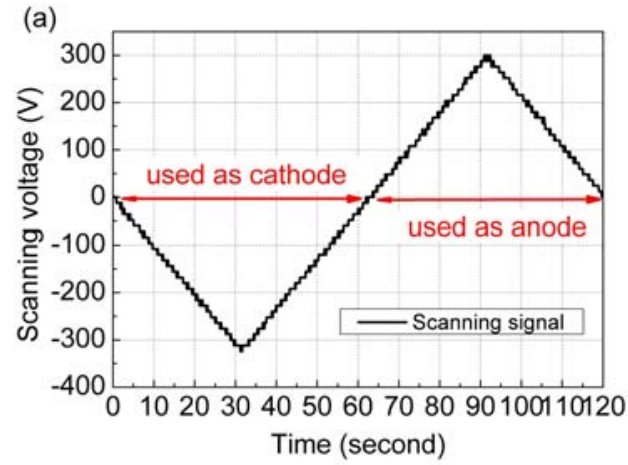

Figure 8(a) 


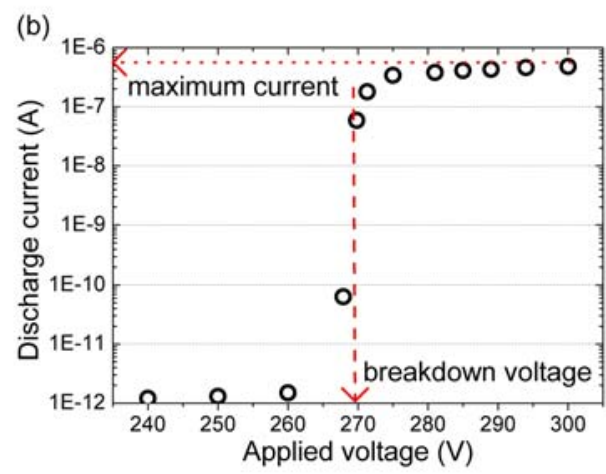

Figure 8(b) 
(c)
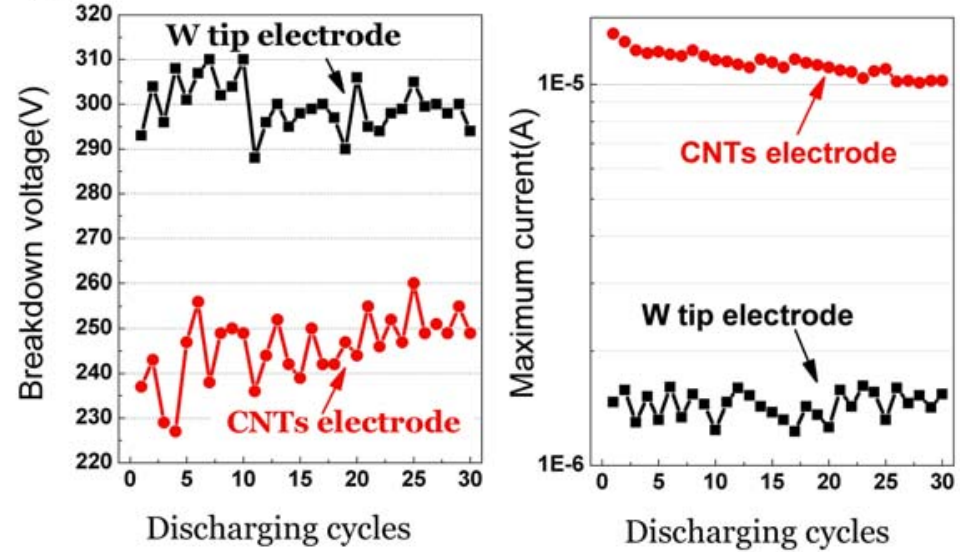

Figure 8(c) 